\title{
Do We Have a Common Mechanism for Measuring Time in the Hundreds of Millisecond Range? Evidence From Multiple-Interval Timing Tasks
}

\author{
Hugo Merchant, Wilbert Zarco, and Luis Prado \\ Departamento de Neurobiología Conductual y Cognitiva, Instituto de Neurobiología, Universidad Nacional Autónoma de México, Campus \\ Juriquilla, Querétaro, Mexico
}

Submitted 5 November 2007; accepted in final form 17 December 2007

\begin{abstract}
Merchant H, Zarco W, Prado L. Do we have a common mechanism for measuring time in the hundreds of millisecond range? Evidence from multiple-interval timing tasks. J Neurophysiol 99: 939-949, 2008. First published December 19, 2007; doi:10.1152/jn.01225.2007. In the present study we examined the performance variability of a group of 13 subjects in eight different tasks that involved the processing of temporal intervals in the subsecond range. These tasks differed in their sensorimotor processing ( $S$; perception vs. production), the modality of the stimuli used to define the intervals ( $M$; auditory vs. visual), and the number of intervals ( $N$; one or four). Different analytical techniques were used to determine the existence of a central or distributed timing mechanism across tasks. The results showed a linear increase in performance variability as a function of the interval duration in all tasks. However, this compliance of the scalar property of interval timing was accompanied by a strong effect of $S, N$, and $M$ and the interaction between these variables on the subjects' temporal accuracy. Thus the performance variability was larger not only in perceptual tasks than that in motor-timing tasks, but also using visual rather than auditory stimuli, and decreased as a function of the number of intervals. These results suggest the existence of a partially overlapping distributed mechanism underlying the ability to quantify time in different contexts.
\end{abstract}

\section{N T R O D U C T I O N}

Organisms have developed different mechanisms to quantify time over a wide range of durations, from microseconds to daily circadian rhythms. It has been suggested that in the middle of these extremes there is a timing mechanism devoted to the hundreds of millisecond scale (Harrington and Haaland 1999; Hazeltine et al. 1997), which is the range of durations used in the present study. Interval timing in this range is a prerequisite in several behaviors, including the perception and production of speech, music, and dance, as well as the performance of sports and estimation of the time that remains before the occurrence of an important event, such as estimating time to contact (Merchant and Georgopoulos 2006).

Different sources of information support the hypothesis of a common timing mechanism in hundreds of milliseconds. First, several psychological studies have shown that the temporal performance follows the scalar property, which defines a linear relationship between the variability of temporal performance and interval duration, in conformity with Weber's law (Matell and Meck 2000). Thus Weber's law is given as $S D(T)=k T$, where $k$ is a constant corresponding to the Weber fraction. In this sense, the coefficients of variation $(\sigma / \mu)$ or the Weber

\footnotetext{
Address for reprint requests and other correspondence: H. Merchant, Departamento de Neurobiología Conductual y Cognitiva, Instituto de Neurobiología, Campus UNAM-UAQ, Juriquilla, Querétaro, Qro 76230, Mexico (E-mail: merch006@umn.edu).
}

fractions show similar values in a variety of tasks and species, suggesting a dedicated temporal mechanism in this time range (Gibbon et al. 1997). For example, in a human discrimination task of time intervals, Getty (1975) described a constant Weber fraction for intervals between 200 and 2,000 ms. Now, another concept in the literature is that the overall variability in a timing task can be dissociated into both time-dependent and time-independent sources of variation (Wing and Kristofferson 1973). Different quantitative and paradigmatic strategies have been used to dissociate the total performance variability. The slope method, for instance, uses a generalized form of Weber's law in which a linear regression between the variability and the squared interval duration is performed. The resulting slope and intercept correspond to the time-dependent and time-independent processes, respectively (Ivry and Hazeltine 1995). It has been demonstrated that the slopes of an interval-discrimination and a repetitive-tapping task are similar for a range of intervals from 325 to 550 ms (Ivry and Hazeltine 1995), again supporting the view of a general interval clock in a variety of contexts. In addition, it has been shown that the temporal variability among individual's performance is correlated between tasks involving explicit timing, such as interval-discrimination and tapping tasks (Keele et al. 1985). This implies that subjects who are good timers in one behavioral context are also good timers in another. In contrast, no such correlations were observed between the acuity on the interval-discrimination task and the performance variability in a circle-drawing task, where subjects used an implicit rather than an explicit timing strategy (Roberstson et al. 1999; Spencer and Zelaznik 2003). Finally, learning studies have demonstrated that intensive training in a timing context can be generalized to other timing behaviors, favoring the common mechanism hypothesis (Buonomano and Karmarkar 2002). For example, training for many days to discriminate time intervals caused an improvement in temporal performance in a motor timing task (Meegan et al. 2000).

The existence of a common timing mechanism implies that the neural substrate of the "internal clock" should be independent of modality, task, and context, as long as the subject's sensorimotor performance requires a carefully timed behavior (Buonomano and Karmarkar 2002; Zelaznik et al. 2002). However, the subjective perception or production of time intervals can be influenced by other factors in addition to actual duration. For instance, temporal performance is more accurate for 1) filled (continuous stimuli) rather than empty intervals (Rammsayer and Lima 1991), 2) auditory rather than visual

The costs of publication of this article were defrayed in part by the payment of page charges. The article must therefore be hereby marked "advertisement" in accordance with 18 U.S.C. Section 1734 solely to indicate this fact. 
stimuli (Goldstone and Lhamon 1974), and 3) multiple isochronous intervals rather than single intervals (Ivry and Hazeltine 1995). Since the interval structure has an important effect on timing sensitivity, the obvious question is whether these factors directly or indirectly modulate the "centralized clock," or whether it is more appropriate to start thinking of more than one timing mechanism (Grondin 2001). Of course, heuristically, the concept of a single clock is simpler and well accepted in the timing community. Indeed, most of the factors that modify the temporal variability have been explained using a conceptual framework where a single timing mechanism is interacting in a context-dependent fashion with a trigger system that defines the onset-offset of time accumulation, as well as with memory and decision-making processes (Matell and Meck 2000; Treisman et al. 1992). Nevertheless, few studies have investigated how different aspects of the interval structure interact simultaneously with the timing performance variability and whether, in this case, the hypothesis of a single timing mechanism still holds.

In the present study we examined the performance variability of a group of 13 subjects in eight different tasks that involved the temporal processing of intervals in the range of 350 to $1,000 \mathrm{~ms}$. However, these tasks differed in their sensorimotor processing, the modality of the stimuli used to define the intervals, and the number of intervals. Different analyses were used to determine the weight of the time-independent parameters of the tasks on the scalar property of interval timing. The results showed a complex interaction between the temporal accuracy of subjects and the sensorimotor processing, modality, and interval number involved in the tasks.

\section{METHODS}

\section{Human subjects}

Thirteen (six male, seven female) subjects, mean (SD) age of 27.2 (2.7) $\mathrm{yr}$ (range: $23-32 \mathrm{yr}$ ), participated in this study. They were right-handed, had normal or corrected vision, and were naive about the task and purpose of the experiment. All subjects volunteered and gave consent for this study before commencement of experiments.
The experiments were approved by the appropriate local ethics committees.

\section{Apparatus}

Subjects were seated comfortably on a chair facing a computer monitor (Dell 19-in. Optiplex) in a quiet experimental room and tapped on a push button (4-cm diameter, Model 7717; Crest, Dassel, $\mathrm{MN}$ ) during the production tasks. In addition, during the perceptual tasks subjects were asked to push a key on the computer keyboard to reflect their decisions. Subjects could not see their hand during tapping. The stimulus presentation and collection of the behavioral responses were controlled by the computer on a custom-made Visual Basic program (Microsoft Visual Basic 6.0, 1998). Auditory stimuli were presented through noise-canceling headphones (MDR-NC50; Sony) and the sampling rate of the push button was $200 \mathrm{~Hz}$.

\section{Task 1: production of multiple time intervals (MTTs)}

EXPERIMENTAL TASK. Subjects were trained to produce tapping movements on a push-button device synchronized to a sensory stimulus and then to continue tapping with the same interval without sensory stimulus (Fig. 1). At the beginning of the trial, the stimuli were presented with a constant interval. Subjects were required to push a button each time a stimulus was presented, which resulted in a stimulus-movement synchronization. After five consecutive synchronized movements the stimulus was eliminated, and the subjects continued tapping with the same interval for four additional intervals. Feedback was displayed on the screen, indicating the human subject's mean intertap interval and SD for the continuation phase of the trial. The interval separating the synchronization and the continuation phase was not included in this feedback measure or in further analyses. The intertrial interval was $1.5 \mathrm{~s}$.

STIMULI. The stimuli were tones $(33 \mathrm{~ms}, 2,000 \mathrm{~Hz}, 50 \mathrm{~dB})$ or visual stimuli in the form of a green square (4- $\mathrm{cm}$ side) presented in the center of a computer screen for $33 \mathrm{~ms}$. The frame rate of the video board $(60 \mathrm{~Hz})$ was accurately calibrated and the duration of visual presentations was controlled precisely in terms of the number of frames. Both the visual and auditory stimuli, although brief, were fully detectable. The interval durations were $350,450,550,650,850$, or

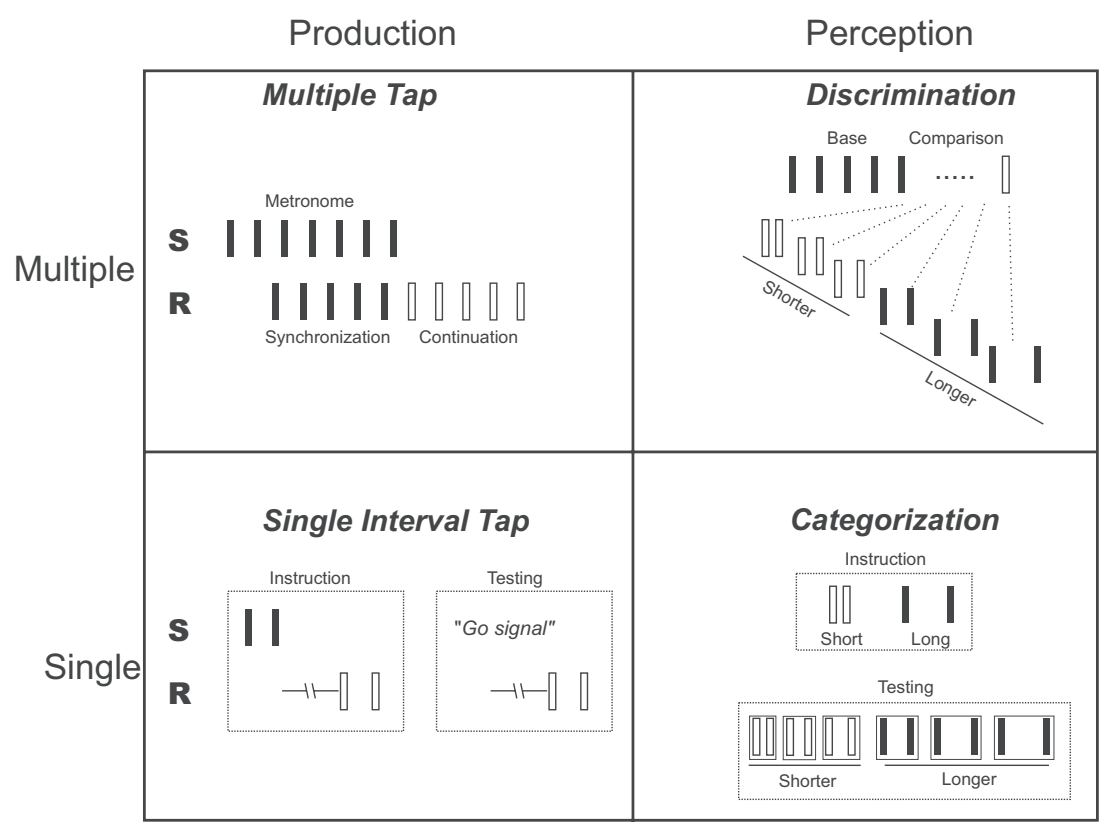

FIG. 1. Timing tasks. This diagram divides 1) production from perception tasks and 2) multiple- from singleinterval tasks. S, stimulus; R, response. 
$1,000 \mathrm{~ms}$. Intervals were chosen pseudorandomly. Ten repetitions were collected for each interval.

\section{Task 2: production of a single time interval (STT)}

EXPERIMENTAL TASK. For each interval there was a training and an execution period (Fig. 1). In the training period, a target interval (two stimuli separated by a particular duration) was presented at the beginning of the trial. Then the subject tapped twice on the push button to produce the same interval. This was repeated for five training trials, after which the subject entered the execution period, where he/she produced another 10 single intervals after a go signal appeared on the screen. Again, feedback was displayed on the screen, indicating the subject's intertap interval and SD across trials of the same interval during the execution period. The intertrial interval was $1.5 \mathrm{~s}$.

STIMULI. The same stimuli and interval durations as those for MTT were used. Ten trials during the execution period were collected for a particular interval duration before changing to another one. The intervals were chosen pseudorandomly.

\section{Task 3: categorization of time intervals (CAT)}

EXPERIMENTAL TASK. The subjects were trained first to press the " $n$ " key on the keyboard after the presentation of an extremely short interval, or to press the " $m$ " key after the presentation an extremely long interval. At least 20 trials (short/long) were performed in this training phase. Categorization feedback was provided during the training phase, with the word "correct" or "incorrect" on the screen. Once the subject learned to associate the short and long intervals with the response on the " $n$ " key and the " $m$ " key, respectively, intermediate intervals were also presented. Thus subjects were required to push one of the keys to indicate their categorical decision for the eight intervals using acquired category boundaries and an implicit standard interval [middle interval (implicit base interval, IBI)] set during the training period (Fig. 1). The intertrial interval was $1.5 \mathrm{~s}$.
STIMULI. The stimuli were tones $(33 \mathrm{~ms}, 2,000 \mathrm{~Hz}, 50 \mathrm{~dB})$ or visual stimuli in the form of a green square (4-cm side), presented in the center of a computer screen for $33 \mathrm{~ms}$. Eight intervals were used for each of the five different implicit intervals. For the 350-ms IBI the intervals were $233,283,316,333,366,383,416$, and 466. For 450-ms IBI the intervals were $299,366,416,433,466,483,533$, and 599. For the $650-\mathrm{ms}$ IBI the intervals were $433,533,583,633,666,699,766$, and 866. For the 850-ms IBI the intervals were 566, 666, 783, 816, $883,916,1,033$, and 1,133. Finally, for the 1,000-ms IBI the intervals were $699,816,933,966,1,033,1,066,1,183$, and 1,299. These intervals were carefully chosen to maximize the quality of the threshold boundaries (Fig. 2). In all cases, the first four were considered short intervals, whereas the last four were long intervals. One repetition of the task for each IBI included the categorization of the eight intervals. The intervals were presented pseudorandomly for each IBI. Ten repetitions were collected for one IBI before moving to the next interval.

SD CALCULATION. The difference threshold is equivalent to one SD from the implicit standard interval (Getty 1975; Keele et al. 1985). To calculate this threshold a psychometric curve was constructed, plotting the probability of long-interval categorization as a function of the interval. A logistic function was fitted to these data and the SD was computed as half the subtraction of the interval at $0.79 p$ and that at $0.21 p$. Details about the logistic function fitting are subsequently given.

\section{Task 4: discrimination of time intervals (DIS)}

EXPERIMENTAL TASK. The subjects were trained to discriminate between a base and a comparison interval, pressing the " $n$ " key on the keyboard for a shorter comparison interval or the " $m$ " key after the presentation of a longer comparison interval. On each trial, participants heard a series of five tones $(33 \mathrm{~ms}, 2,000 \mathrm{~Hz}, 50 \mathrm{~dB})$ or viewed five visual stimuli (green squares, $10-\mathrm{cm}$ side, $33 \mathrm{~ms}$ ). The first five created the three isochronous base intervals. The sixth one produced the comparison interval that was either shorter or longer than the base (Fig. 1). Again, 10 trials (extreme short/long) were performed in the
A

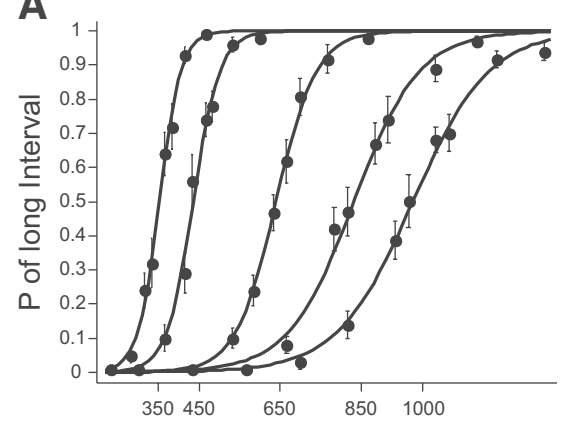

B

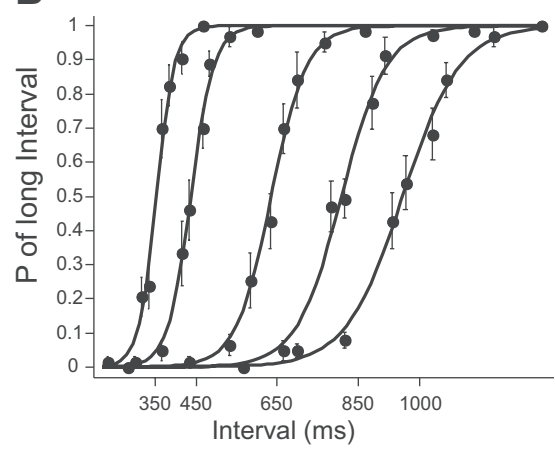

Visual
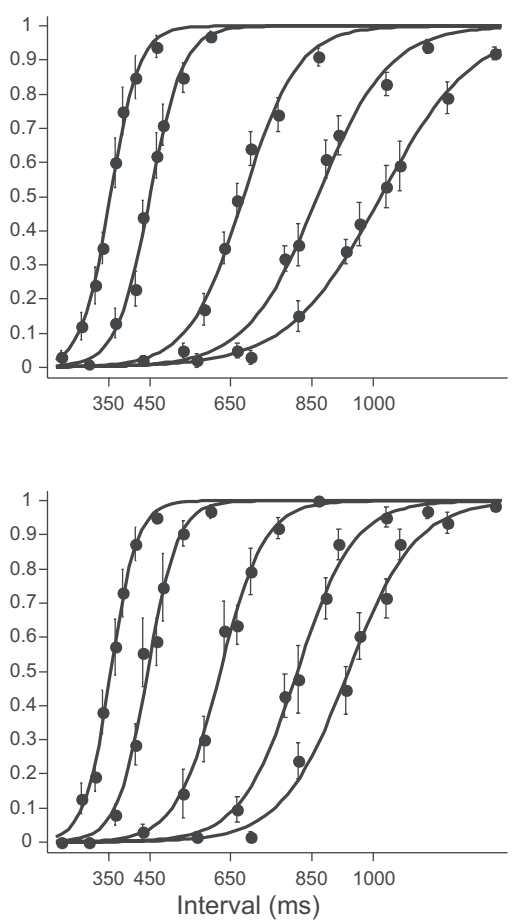

FIG. 2. A: psychometric functions (means $\pm \mathrm{SE}$ ) for the categorization task using auditory and visual markers. $B$ : psychometric functions for the discrimination tasks. The probability of long-interval categorizations or discriminations is plotted as a function of the interval. Logistic functions are fitted to the data. 
training phase, followed by 8 trials for each of the eight base/ comparison combinations. Feedback was provided, with the word "correct" or "incorrect" on the screen during the training phase. The intertrial interval was $1.5 \mathrm{~s}$.

STIMULI. The intervals used in the categorization task were also used in this task as comparison for each of the five different base intervals $(350,450,650,850$, and $1,000 \mathrm{~ms})$. One repetition of the task for each base interval included the discrimination of the eight intervals, and eight repetitions were collected. In addition, in $20 \%$ of the trials the base and comparison intervals were chosen at random within the range of 330 to $1,100 \mathrm{~ms}$. This was done with the purpose of maintaining the subject's attention to both interval durations across all trials. Finally, the comparison intervals were presented pseudorandomly within each base interval and the order between base intervals was chosen randomly.

\section{SD calculation}

The SD was calculated in the same fashion as in the categorization task.

\section{Timing task procedure}

The subjects performed the tasks in random order in four sessions. At least eight repetitions were collected for each condition and task. Before data collection, practice trials were given in the four tasks until the subjects acknowledged that they understood the tasks and were comfortable with their performance.

\section{Analysis of behavioral data}

GENERAL. Standard statistical techniques were used for data analysis including $t$-test, repeated-measures ANOVA, Pearson correlation, and linear regression (Snedecor and Cochran 1989). The reported $P$ values in the repeated-measures ANOVAs correspond to the Greenhouse-Geisser test, which corrects for possible deviations in sphericity. The level of statistical significance to reject the null hypothesis was $\alpha=$ 0.05. Subroutines written in Matlab and the SPSS statistical package (version 12 2003, SPSS, Chicago, IL) were used for the statistical analyses.

LOGISTIC REGRESSION. This regression was used for the psychometric data and was given by

$$
y=\frac{\left(p_{1}-p_{4}\right)}{1+\left(\frac{x}{p_{3}}\right)^{p_{2}}}+p_{4}
$$

where $p_{1}$ and $p_{4}$ correspond to the maximum and minimum values of $y, y$ is the probability of long interval categorization, $p_{2}$ is the estimated slope, and $p_{3}$ corresponds to the value of $x$ (time interval) at half of the maximum value of $y$. The percentage of variance explained $\left(R^{2}\right)$ was $>90 \%$ in all the fittings.

SLOPE ANALYSIS. A linear regression between the timing variance $\left(\sigma^{2}\right)$ and the interval duration squared $\left(D^{2}\right)$ was performed (see $E q .3$ of Ivry and Hazeltine 1995)

$$
\sigma^{2} \text { Total }=k^{2} D^{2}+c
$$

where $k$ is the slope that approximates the Weber fraction and $c$ is a constant representing the time-independent variability component.

\section{RE S ULTS}

In the present study 13 subjects performed eight timing tasks: two motor [single (STT) and multiple (MTT) interval tapping] and two perceptual [categorization (CAT) and discrimination (DIS)] paradigms in which the intervals were defined by auditory (A) or visual (V) stimuli (Fig. 1). All the tasks involved temporal processing of intervals in the range of 350 to $1,000 \mathrm{~ms}$; however, they also covered a wide range of behavioral contexts, differing in their perceptual, decisionmaking, memory, and execution requirements. Indeed, besides the different modalities and the perceptual- versus motortiming comparison, these tasks can also be grouped by their cyclic nature into single- or multiple-interval paradigms (Fig. 1). The single-interval tasks (STT and CAT) had two important phases: a training phase where the subjects set an implicit interval and a test phase were they used this implicit interval to solve the tasks. In contrast, during the multiple-interval tasks (MTTs and DIS) there was not only a periodic temporal processing of one interval, but also a working-memory component used to execute the tasks. Thus a great advantage of the present database is that the subjects performed all the tasks, and the temporal and nontemporal components of their behavior were compared with high sensitivity within and between subjects.

\section{Psychometric performance in the perceptual tasks}

The psychometric curves for the CAT (Fig. 2A) and DIS (Fig. 2B) tasks were sigmoidal and well suited to measure temporal acuity, with correct responses in the extremely short and long intervals, and decision errors in the intermediate intervals. Then, with the method of constant stimuli used here, the thresholds were properly estimated (Getty 1975). It is important to mention that similar results were obtained using a staircase psychometric method (data not shown; Wright et al. 1997).

\section{Distributions of the temporal performance across tasks}

Figure 3 shows the distributions of internally generated tap intervals in the MTT and STT tasks, as well as the mean distributions for the perceived implicit or base intervals in the CAT and DIS tasks, respectively. Of course in the perceptual tasks the depicted distributions correspond to the theoretical "interval representations" (Bassalou et al. 1998) extracted from the psychometric performance of the subjects. Thus the mean of perceptual distributions corresponded to the point of subjective equality: the interval at $0.5 p$ in the psychometric curve (see Fig. 2). Accordingly, the SD was computed as half the difference between the interval at $0.79 p$ and the interval at $0.21 p$ in the psychometric curve (Keele et al. 1985).

It is evident that the distributions showed an increase in SD as a function of the interval and that the mean and variability were different across tasks and sensory modalities. Using the data depicted in Fig. 3 we carried out detailed analyses of the variability of temporal performance for each of the eight task conditions.

\section{Temporal variability}

Figure 4 shows that the temporal variance increased as a function of the interval squared in all tasks, following the scalar property of interval timing. However, this relation was different across tasks and modalities. A repeated-measures 

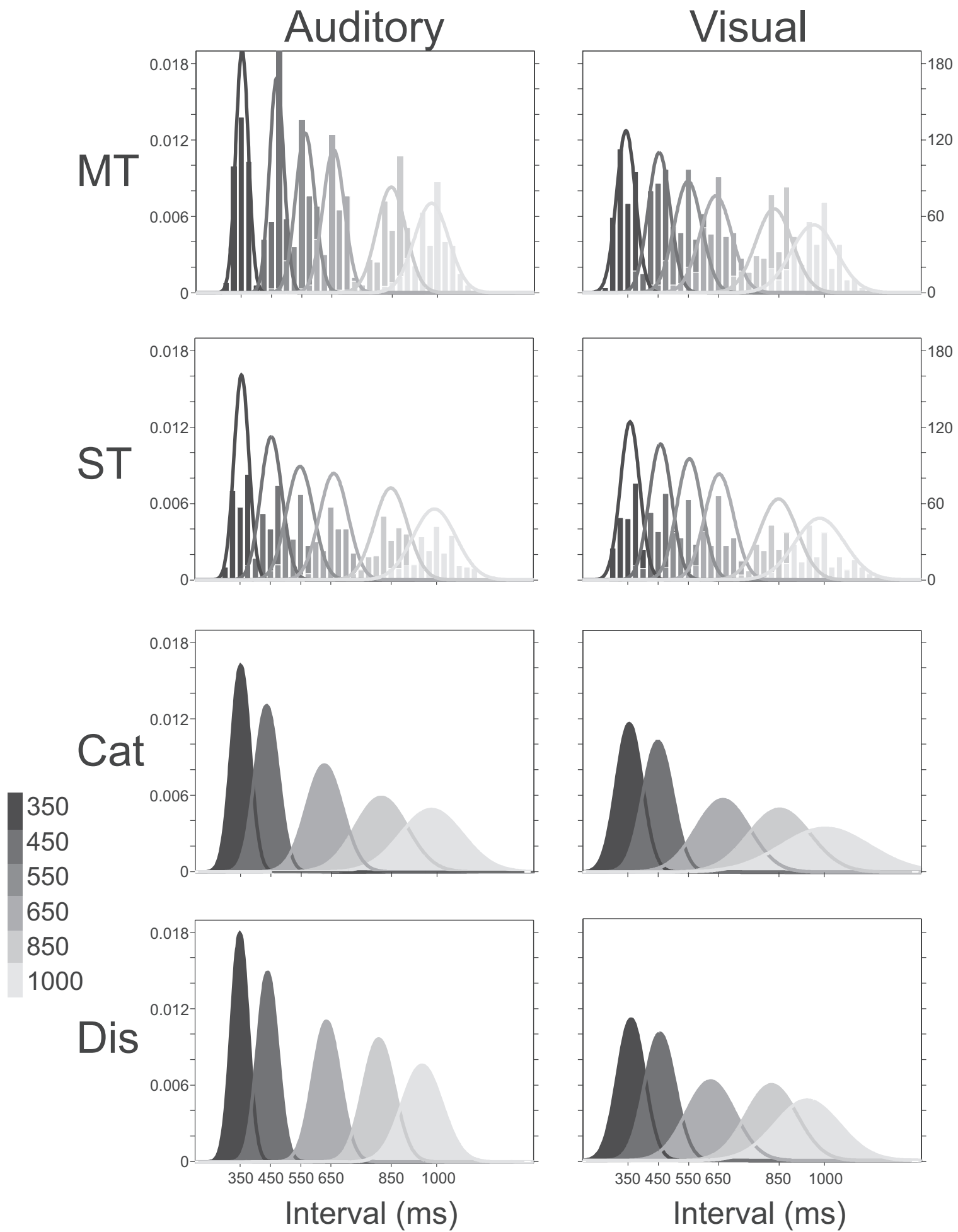

FIG. 3. Distributions of internally generated tap intervals in the multiple-tap task (MTT) and single-tap task (STT), as well as the mean distributions for the perceived implicit or base intervals in the categorization (CAT) and discrimination (DIS) tasks, respectively. Both auditory and visual conditions are shown. The interval durations are grayscale coded (cf. bottom left). The right axes in MTT and STT represent frequency of tapped intervals.

ANOVA was carried out using the variance as dependent variable and the target interval, task, and modality as the within-subject factors. The results showed significant main effects for target interval $\left[F_{(4,48)}=25.5, P<0.0001\right]$, task $\left[F_{(3,36)}=16.3, P<0.0001\right]$, and sensory modality $\left[F_{(1,12)}=\right.$ 16.6, $P=0.002]$. In addition, all the paired interactions also showed significant effects: the interval $\times \operatorname{task}\left[F_{(12,144)}=9.4\right.$, $P<0.0001]$, the interval $\times$ modality $\left[F_{(4,48)}=5.2, P=\right.$ $0.018]$, and the task $\times$ modality interactions $\left[F_{(3,36)}=6.2, P=\right.$ $0.012]$. These results indicate that the variance differed across intervals and that this change was dependent on task and modality. 


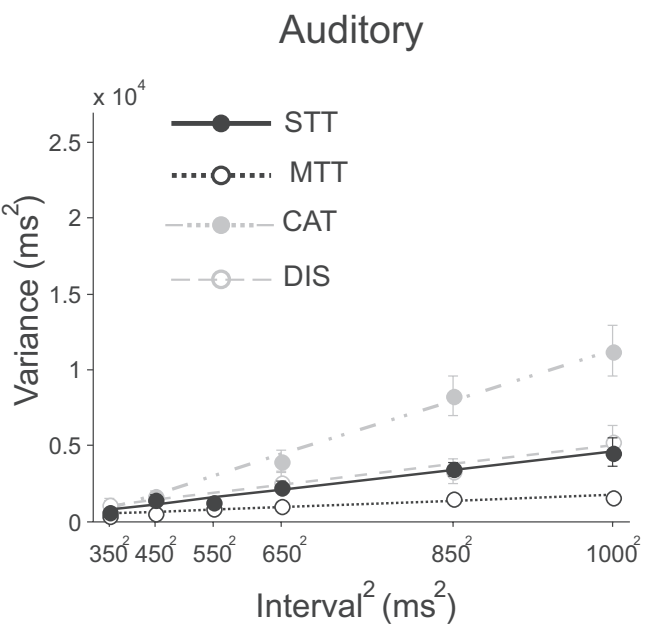

Slope analysis

We used the slope method to analyze further the temporal variance across tasks. This analysis assumes that the total variability in a timing task can be decomposed into the variability associated with the timing mechanisms and the variability resulting from duration-independent processes. The slope method uses a generalized form of Weber's law in which a linear regression between the variability and the squared interval duration is performed. The resulting slope and intercept correspond to the time-dependent and time-independent processes, respectively (Ivry and Hazeltine 1995). The main objective here was to test whether the time-dependent variability (slope) differed between the eight tasks. Table 1 shows the intercept and slope (mean and SE across subjects) for all the timing tasks. It is clear that both measures were different between tasks and modalities. Nevertheless, the percentage of variance accounted for in these linear fittings was quite high across tasks (Table 1).

Focusing on the time-dependent variability, we performed a repeated-measures ANOVA where the slope was used as dependent variable and the sensorimotor context $[S$ (perception vs. production)], the modality [ $M$ (auditory vs. visual)], and the number of intervals $[N$ (one or four)] as within-subject factors. The results showed significant main effects for $S\left[F_{(1,12)}=\right.$ 22.9, $P<0.0001], N\left[F_{(1,12)}=14.3, P=0.003\right]$, and $M\left[F_{(1,12)}=10.1, P=0.008\right]$. In addition, the $S \times M$ $\left[F_{(1,12)}=7.1, P=0.021\right]$ and the $S \times N$ interactions $\left[F_{(1,12)}=\right.$ $5.9, P=0.031]$ also showed significant effects. However, the $M \times N$ interaction $\left[F_{(1,12)}=1.7, P=0.215\right]$ was not signif-

TABLE 1. Intercept (c), slope $\left(k^{2}\right)$, and $R^{2}$ (mean $\pm S E$ ) from the slope analysis for all tasks

\begin{tabular}{lccc}
\hline \hline Task & $c$ & Slope & $R^{2}$ \\
\hline MTT-A & $317.8 \pm 92.3$ & $0.00142 \pm 0.0001$ & $0.71 \pm 0.066$ \\
STT-A & $245.8 \pm 184.1$ & $0.00437 \pm 0.0009$ & $0.68 \pm 0.068$ \\
CAT-A & $-630.7 \pm 336.6$ & $0.0119 \pm 0.0016$ & $0.79 \pm 0.047$ \\
DIS-A & $476.5 \pm 301.9$ & $0.00454 \pm 0.001$ & $0.64 \pm 0.088$ \\
MTT-V & $427.1 \pm 184$ & $0.00265 \pm 0.0007$ & $0.60 \pm 0.075$ \\
STT-V & $245.8 \pm 225$ & $0.00485 \pm 0.0003$ & $0.74 \pm 0.039$ \\
CAT-V & $-2,065.2 \pm 1,151$ & $0.0258 \pm 0.006$ & $0.77 \pm 0.048$ \\
DIS-V & $1,799.1 \pm 1,190$ & $0.0109 \pm 0.02$ & $0.63 \pm 0.095$ \\
\hline
\end{tabular}

Values are means \pm SE. A, auditory; V, visual.
Visual

FIG. 4. Mean variances plotted as a function of the interval duration squared for the 4 different tasks in the auditory and visual conditions. icant. Thus the slope was larger for perceptual than for production tasks [ $t$-test, $t(51)=4.83, P<0.0001]$ and larger for the visual than for the auditory modality [ $t$-test, $t(51)=-3.18$, $P=0.003]$. Furthermore, a $t$-test between the tasks with single (CAT-STT) versus multiple (DIS-MTT) intervals, showed a significantly larger slope in the former than in the latter $[t(51)=3.95, P<0.0001]$. Therefore the present results revealed a complex interaction between the scalar property of interval timing and the context in which the subjects temporalized their behavior, ranging from very small slopes in the MTT-A to very large slopes in the CAT-V.

The significant $S \times M$ interaction in the preceding ANOVA indicates an interesting aspect of the timing performance when comparing our tasks: the increase in slope between the auditory and visual modalities was more pronounced in the perceptual than in the production tasks. In fact, a specific paired-samples $t$-test revealed that the slope difference between the visual and auditory modalities was statistically larger in the perceptual [difference: $0.042 \pm 0.05$ (mean \pm SD)] than in the motor timing tasks [difference: $0.005 \pm 0.03 ; t(25)=3.28, P=$ 0.003]. Thus these results suggest that, besides the fact that there was a generalized increase in variability when the time intervals were defined by visual rather than auditory stimuli, the increase in the Weber fraction due to visual stimuli was more important in perceptual than in motor contexts. Likewise, the significant $S \times N$ interaction in the ANOVA indicates that the decrease in slope in tasks with multiple intervals was more pronounced in perceptual than in production paradigms. Indeed, the slope difference between single and multiple intervals was significantly larger in the perceptual (difference: $0.011 \pm 0.016)$ than in the motor timing tasks [difference: $0.0026 \pm 0.03 ; t(25)=2.49, P=0.02]$.

Overall, these results suggest that, although the generalized form of Weber's law adequately captured the performance variability for all the tasks, the time-dependent element of the model (slope) differed between the perception/production, the visual/auditory, and the single-/multiple-interval dimensions. These results can have at least two interpretations: the timing mechanism is different across these dimensions or the timing mechanism is modulated directly or indirectly by $S, N$, and $C$. In this regard, it is important to consider the possibility that slope is a measure that represents not only the time-dependent source of variation, but also some other behavioral elements 
that are present in the timing paradigms. In fact, Ivry and Hazeltine (1995) emphasized the importance of performing the slope analysis under very similar task conditions.

Finally, we performed two additional repeated-measures ANOVAs, where the intercept and the $R^{2}$ of the slope analysis were used as dependent variables and $S, M$, and $N$ were the within-subject factors. The results showed that only $N$ showed significant main effects in both the intercept $\left[F_{(1,12)}=7.6, P=\right.$ $0.017]$ and the $R^{2}\left[F_{(1,12)}=8.5, P=0.013\right]$ ANOVAs. The remaining factors and all the interactions showed no significant effects in these analyses. Therefore these results indicate, first, that the intercept was not affected in the same way as the slope by the different behavioral contexts. Second, the time-independent sources of variance (the intercept) were significantly lower for the CAT and STT than for the DIS and MTT, which is evident in Table 1. Thus the sensory processing, motor execution, and memory requirements for single-interval tasks are probably less demanding than those for multiple-interval timing tasks. In the case of CAT, the intercept showed negative values, which is an indication that the generalized Weber law is probably not the best model to explain the temporal variability as a function of duration in the intervals tested (Ivry and Hazeltime 1995). Nevertheless, we tested other different regression models (i.e., SD vs. Interval) for CAT and the resulting intercept was even more negative. On the other hand, the linear fittings in the single-interval tasks were significantly higher than those in DIS and MTT. However, it is important to state that all the fittings were significant (Table 1) and thus is not feasible that the reported slope differences among tasks were mainly due to a poorer fit in the linear regression between timing variance and the interval duration squared.

\section{Correlation analysis}

It has been reported that individual differences in the variability of a tapping task are correlated across different effectors (Keele et al. 1985). Furthermore, a significant SD correlation between production and perception tasks has also been documented (Spencer and Zelaznik 2003). This means that subjects that are good timers in one behavioral context are good timers in another one. Thus the existence of significant intrasubject correlations in the temporal variability across different timing tasks has been taken as an indication of a common timing mechanism.

Consequently, here we exploited individual variation in temporal performance to determine whether there was a common timing mechanism across the eight tasks. Correlations were carried out on the Z-scored timing SD to analyze the precision of individual subjects between pairs of tasks for all interval durations. The Z-score values allowed for comparisons of the subject's timing precision without including the effect of the scalar property of interval timing in the correlation. The results are depicted as a correlation matrix in Fig. 5, where it is evident that several tasks showed significant pairwise correlations [Bonferroni correction: $P<0.0013(P=0.05 / 36$ comparisons)]. Generally, the individual differences were more consistent between the same tasks and different modalities. For instance, the subjects' SD correlation across visual and auditory stimuli in the CAT task had an $R=0.47$, which is a high correlation for 13 subjects using the performance during the five interval durations.

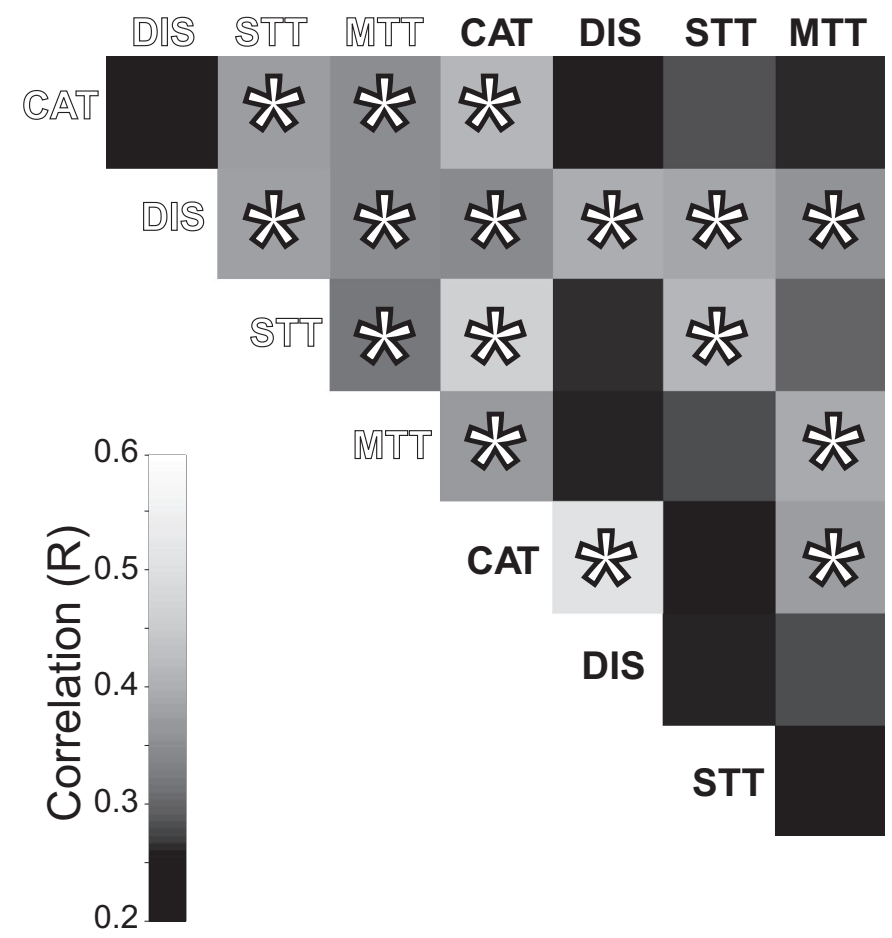

FIG. 5. Correlation matrix showing the Pearson $R$ value in a grayscale (inset, bottom left) for all possible pairwise task comparisons. Asterisks indicate significant correlations $(P<0.05)$ between specific task pairs. Open and closed fonts correspond to tasks with auditory and visual markers, respectively.

Even if some pairwise comparisons did not show significant effects, in general, the CAT showed significant correlations with the STT, the DIS with the MTT, the STT with the MTT, and finally the DIS with the CAT. Thus correlation analysis shows a complex picture of task associations where, again, the behavioral dimensions of modality, number of intervals, and sensorimotor processing played important roles in determining the level of task correlation. Of course, these data can be interpreted again in two ways: the notion of a common timing mechanism that is modulated by these three behavioral dimensions or the idea of multiple timers that are activated in a context-dependent fashion.

\section{Principal-component analysis}

The correlation matrix in Fig. 5 depicts a complex multidimensional interaction between the tasks' variability. Therefore to determine the number of dimensions that better explained such interactions we performed a principal-component analysis (PCA) on the subjects' Z-scored timing SD across tasks. PCA is a mathematical procedure that allows for the reduction dimensions in a data set with multiple parameters. This method generates a new set of variables, called principal components (PCs). Each PC is a linear combination of the original variables, so that the PCs are orthogonal to each other. The first PC accounts for as much of the variability in the data as possible and each succeeding PC accounts for as much of the remaining variability as possible. Thus PCA extracts the most meaningful driving principles governing the behavior of a multidimensional data set.

The percentage of variance explained by the different PCs (from 1 to 8 ) on the correlation matrix (Fig. 5) are the 
following: 42.6, 13.3, 12.7, 10, 7.5, 5.7, 4.9, and 3.3. It is evident that the only clear break in the amount of variance accounted for by each component was between the first and second components. However, the first PC explained $<45 \%$ of the variance, when commonly the first PC explains $>60 \%$ of the variance. Therefore the PCA indicates that more than one dimension is needed to explain the interaction in timing variability between our eight tasks. In fact, following both the Kaiser criterion (Kaiser 1960), which considers PCs only with eigenvalues $>1$, and the scree test (Cattell 1966) we retained the first three PCs as important components. Thus the first three PCs explained roughly two thirds of the total variability $(68.6 \%)$. Consequently, it is reasonable to suggest the existence of a distributed timing mechanism that depends on the interaction of different components (i.e., $S, N$, and $M$ ). Nevertheless, it is important to emphasize that this analysis was performed with the purpose of determining the number and not the identity of variables explaining the multidimensional interactions in the temporal variability between our eight tasks.

\section{Multiple linear models}

The preceding correlation and PCA analyses suggested a complex interaction between tasks, where the $S, N$, and $M$ had an important role in the level of association among paradigms. On the other side, the slope analysis indicated that the Weber fractions were different across tasks and that these parameters modulated the magnitude of the slope. Thus overall these findings lean toward a distributed rather than a centralized timing mechanism. However, a distributed system could have at least two configurations, consisting of totally independent or partially overlapping variables. We designed two multiple linear regression models to test which configuration could better explain the variability between tasks. The partially overlapping distributed (POD) model integrated the information about the scalar property of interval timing with the influence of the three other behavioral dimensions $(S, N, M)$ across tasks (Merchant et al. 2004). The hypothesis behind this analysis is that the total task variability can be decomposed in the timing variability plus the variability related to the interaction between the timing and the other three behavioral dimensions. The POD model is expressed as

$$
\begin{aligned}
\sigma^{*}=b_{0}+b_{1} D+b_{2} D S+b_{3} D N+b_{4} D M & +b_{5} D S M \\
& +b_{6} D S N+b_{7} D N M+b_{8} D S N M
\end{aligned}
$$

where $\sigma^{*}$ is the log-transformed timing $\mathrm{SD}, b_{0}-b_{8}$ are regression coefficients, $D$ is the interval duration, $S$ is the sensorimotor context (perception $=1$, production $=0), N$ is the number of intervals ( 1 or 4 ), and $M$ is the modality (auditory $=1$, visual $=0$ ). The $\log$ transformation was performed on the SD to correct for heteroscedasticity (Draper and Smith 1981). However, it is important to mention that the results of the two models (Eqs. 3 and 4) were very similar with or without the log transformation.

On the other hand, the totally distributed (TD) model assumed that the task variability was the sum of independent sources of variance associated with each task. Thus the model is expressed as

$$
\sigma^{*}=b_{0}+b_{1} t_{1}+b_{2} t_{2}+b_{3} t_{3}+b_{4} t_{4}+b_{5} t_{5}+b_{6} t_{6}+b_{7} t_{7}
$$

where $\sigma^{*}$ is the log-transformed timing SD; $b_{0}-b_{7}$ are regression coefficients; and $t_{1}-t_{7}$ are the tasks in the following incremental order: CAT-A, CAT-V, DIS-A, DIS-V, STT-A, STT-V, and MTT-A. Since each task was a dummy variable with a value of 0 or 1, the last task (MTT-V) was the reference task and thus was not included explicitly in the model.

The adjusted $R^{2}$ was computed to compare the explanatory power of two models with different degrees of freedom (Zar 1998). The results showed that the adjusted proportion of variance accounted for was considerably larger for the POD of $E q .3$ (adjusted $R^{2}=0.504$ ) than that for the TD model of $E q .4$ (adjusted $R^{2}=0.209$ ). Nevertheless, both regression models were significant $\left[F_{(8,519)}=67, P<0.0001\right.$ for the former; $F_{(7,512)}=20.5, P<0.0001$ for the latter]. Overall, the results suggest that a partially overlapping mechanism for temporal processing is the best candidate. Neither a totally centralized nor a totally distributed mechanism seemed appropriate, as indicated by the slope and multiple regression analyses, respectively. Then, the partially overlapping mechanism may include a core of brain areas involved in the underpinnings of the scalar property and a set of other areas interacting with the core in a context-dependent fashion (see DIScussion).

Finally, the standardized regression coefficients of the POD were rank-ordered to determine the relative explanatory power of each parameter in the model. The results of this analysis are shown in Table 2, where it is evident that the interval duration was the most important parameter, followed by the DS, DM, DSM, DNM, and DS interactions. Thus the results showed that the scalar property was the most prevalent parameter for explaining the total performance variability across tasks. In addition, these results indicate that the interaction between timing variability and the other three parameters had a prominent effect, corroborating the importance of $S, N$, and $M$ in the subject's performance across tasks.

\section{DIS C US S ION}

This study confirmed the presence of the scalar property of interval timing in eight different tasks that required temporal processing in the subsecond range. Nevertheless, all the performed analyses suggested that $S, N$, and $M$ and the interaction between these parameters had a critical effect on the temporal accuracy of subjects studied across all these conditions.

TABLE 2. Rank-ordered standardization coefficients for the different parameters of the POD model of Eq. 3

\begin{tabular}{lrrr}
\hline \hline \multicolumn{1}{c}{ Variable } & $\begin{array}{c}\text { Standardized } \\
\text { Coefficient }\end{array}$ & $t$-Value & $P$ \\
\hline $\mathrm{D}$ (main effect) & 0.7103 & 17.0037 & 0.0000 \\
$\mathrm{D} \times \mathrm{S}$ & -0.4597 & -7.0335 & 0.0000 \\
$\mathrm{D} \times \mathrm{M}$ & -0.4317 & -6.6058 & 0.0000 \\
$\mathrm{D} \times \mathrm{S} \times \mathrm{M}$ & 0.2010 & 2.6051 & 0.0095 \\
$\mathrm{D} \times \mathrm{N} \times \mathrm{M}$ & 0.1957 & 2.5358 & 0.0115 \\
$\mathrm{D} \times \mathrm{N}$ & 0.1327 & 2.0312 & 0.0427 \\
$\mathrm{D} \times \mathrm{S} \times \mathrm{N}$ & 0.0533 & 0.6901 & 0.4905 \\
$\mathrm{D} \times \mathrm{S} \times \mathrm{N} \times \mathrm{M}$ & -0.0625 & -0.7575 & 0.4491 \\
\hline
\end{tabular}




\section{Scalar property}

Experimental psychologists have focused more on the variability of temporal processing than on the time-constant error. This is due, in large part, to the ubiquitous scalar property of interval timing, which is a form of Weber's law. The scalar property has been observed in many timing tasks and species. In addition, the scalar property is not followed by subjects with timing deficiencies, such as Parkinson's disease patients (Artieda et al. 1992; Merchant et al. 2007). Thus similar Weber fractions across timing contexts and durations have justified the view of a unified mechanism of temporal processing (Gibbon et al. 1997). Nevertheless, some empirical demonstrations have revealed that the Weber fraction is not always homogeneous with a maximal sensitivity at particular interval durations (Drake and Botte 1993; Fetterman and Killeen 1990; Grondin 1992). Thus even the support of Weber's law for a central timing mechanism has some exceptions.

On the other hand, a number of information-processing models have been proposed based on the scalar property. All these models include clock, memory, and decision components and can account for human and animal timing performance in a variety of tasks (Matell and Meck 2000). However, apart from their heuristic value, it is very difficult to translate the psychological mechanism of such models into a feasible neurophysiological substrate of interval timing (Buhusi and Meck 2005). In fact, all functional magnetic resonance imaging (fMRI) studies have shown that temporal processing is performed by a distributed system of brain areas (Harrington and Haaland 1999; Rao et al. 2001). Consequently, these neurophysiological findings argue against the hypothesis of a centralized timing mechanism. The contemporary consensus suggests that interval timing is ingrained in basic sensorimotor processes (Buonomano and Karmarkar 2002; Penney 2003).

The present study found that the linear increase in variability as a function of interval duration was modulated by the three nontemporal parameters of the tasks. From the central mechanism point of view, it could be argued that these parameters have a differential impact on the memory, decision, or motor components of the tasks. In contrast, the distributed hypothesis will support the notion of different neural circuits engaged in time processing according to $S, N$, and $M$ (Karmarkar and Bounomano 2007). Since our correlation and multiple regression analyses indicate a common relation between some tasks, we suggest an intermediate hypothesis: a large distributed system is devoted to temporal processing in different circumstances, but the processing weight of the different nodes of the distributed system will change according to the task contingencies. Recent fMRI studies are starting to describe the structures involved in different time-processing contexts (Lewis and Miall 2003). Thus our hypothesis is testable using different imaging and neurophysiological techniques and can account for most of the previous timing literature.

\section{Perception versus production}

The slope and multiple regression analyses showed that the sensorimotor context was the most important nontemporal parameter affecting the subjects' performance. In contrast, the correlation analysis showed that the individual pattern of variability was similar between some perceptual and production tasks. These contradictory results can be explained considering a partially overlapping neural network. In this sense, Lewis and Miall (2003) carefully grouped the fMRI timing literature on the basis of the different task parameters used. They found two main networks: 1) an "automatic" timing system that is primarily involved in the continuous measurement of predictable, subsecond intervals defined by movement; and 2) a "cognitively controlled" timing system that is more involved in the measurement of suprasecond intervals not defined by movement and occurring as discrete epochs. The former depends on motor circuits including the motor cortex and the dorsal and ventral premotor areas, whereas the latter involves prefrontal and parietal regions. The production tasks used in the present study can be clearly included in the automatic timing system. In contrast, the perception tasks can be included in the cognitive timing system, but with less certainty, since these tasks were also in the subsecond range. Nevertheless, it has been shown that the parietal cortex is engaged in time processing during a discrimination task (Leon and Shadlen 2003), supporting the hypothesis that perception tasks depend on the "cognitive" timing system.

On the other hand, recent studies have shown that the supplementary motor area (SMA) and the basal ganglia are probably the main overlapping elements of the timing network. These areas are activated in production and perception tasks using a variety of interval structures (Macar et al. 2002, 2006; Meck and Benson 2002; Pouthas et al. 2005; Rao et al. 1997, 2001; Schubotz et al. 2000). Thus the significant correlations between individual performance in perceptual and production tasks could be due to the prevalence of these areas during time-processing behaviors.

\section{Number of intervals}

In both perceptual and production tasks, it has been demonstrated that temporal variability decreases as a function of the number of intervals to be timed (Ivry and Hazeltine 1995; Keele et al. 1989). For example, Schultze (1989) found that the difference limen decreased as a function of the number of base intervals in a discrimination task. Here we found that tasks involving temporal processing of multiple intervals decreased in performance variability with respect to single-interval tasks. In addition, this decrease was more pronounced in perceptual than in production tasks, suggesting that the strength of the reference interval representation also depends on the context in which the multiple intervals are processed. From the perspective of a distributed network, the decrease in variability with the number of timed intervals could be related to a change in activity within and between the different nodes of the system and/or the participation of other areas. In this sense, it has been reported that the cerebellum is activated during discrete but not during continuously timed movements (Spencer et al. 2007). To our knowledge, however, no fMRI study has investigated the neural networks activated during single- and multipleinterval timing tasks.

\section{Modality dimension}

The sensory modality used to define the intervals is an important parameter for inducing major changes in temporal processing. In both perceptual and production tasks, visual 
stimuli produce more variable time estimates than auditory ones (Goldstone et al. 1959; Repp and Penel 2002; Wearden et al. 1998). In discrimination tasks this applies not only to filled intervals but also to empty intervals (Grondin et al. 1998). Our present results corroborate that timing variability is larger with visual than with auditory markers. In fact, the slope analysis showed that the time-dependent source of variation was larger in the visual than that in the auditory condition. In contrast, the correlation analysis showed that the individual performance was more consistent between the same tasks using visual or auditory stimuli to define the intervals. These findings can be explained if we consider that sensory markers are probably processed at the initial processing stages of the hypothetical distributed timing system, whereas the remaining aspects of a specific timing task may engage similar and larger parts of this distributed system. Thus even if the temporal variability is larger in visual than that in the auditory condition, the overall performance of the subjects is quite consistent within the context of a given task. Favoring this notion are fMRI studies that reported that early and late visual or auditory areas are activated during production and perception tasks of intervals using visual or auditory markers, respectively (Jancke et al. 2000; Jantzen et al. 2005; Schubotz et al. 2000). In addition, these studies have found that a larger set of areas was activated in both sensory conditions, including SMA, dorsal premotor cortex, posterior parietal cortex, putamen, and the cerebellum.

Now, the picture becomes more complicated when we consider that the increase in variability for intervals defined by visual rather than auditory stimuli was more pronounced in perceptual than in motor contexts. The interaction between these nontemporal parameters is probably due to the wellknown network division of visual processing for perception and action (Goodale and Milner 1992). Thus the parietofrontal system is specialized in the visual control of action, whereas the occipitotemporal system is dedicated to the perception of the visual world. Support for the perception-action hypothesis has come from experiments showing that visually guided actions are largely refractory to perceptual illusions (Goodale and Westwood 2004). Under this scenario the network engaged in motor timing probably has direct access to visual information through the efficient parietofrontal system, resulting in a small difference in temporal processing between modalities. In contrast, the perceptual timing network, which may include parietal and prefrontal areas, could have indirect access to visual information though the occipitotemporal visual pathway, explaining why the temporal variability in the perceptual timing tasks was considerably larger with visual rather than auditory markers.

\section{Conclusions}

Allan and Kristofferson (1974) argued that the independence of temporal judgments from sensory characteristics supports the idea of a central timing mechanism. Here, on the contrary, we found that the $S, N$, and $M$ and their interactions had an important effect on the subjects' temporal accuracy. Thus the present results support the notion of a distributed system for time processing. Neurophysiological evidence for the existence of task-dependent timing processes has come from recent fMRI research, demonstrating that the specific neural structures re- cruited for temporal processing may be at least partially determined by the way in which timing information is presented (Jantzen et al. 2004). Thus different nodes of the distributed system may change their level of participation in the network depending on nontemporal parameters such as $S, N$, and $M$. In addition, structures such as SMA and the basal ganglia could be the core of this network, participating in most of the timing contexts. These last areas may be closely related to the expression of the scalar property in different timing tasks.

\section{A C K N OW LED G M EN T S}

We thank A. Georgopoulos and M. Merzenich for helpful discussions on the project; J. Ashe, B. Averbeck, G. Baud-Bovy, and G. Pellizzer for fruitful comments on the manuscript; D. Pless for proofreading the manuscript; and R. Paulín for technical assistance.

\section{G R A N T S}

This work was supported in part by Programa de Apoyo a Proyectos de Investigación e Innovación Tecnológica Grant IN209305 and Fogarty International Research Collaboration Award Grant TW007224-01A1.

\section{REFERENCES}

Allan LG, Kristofferson AB. Psychophysical theories of duration discrimination. Percept Psychophys 16: 26-34, 1974.

Artieda J, Pastor MA, Lacruz F, Obeso JA. Temporal discrimination is abnormal in Parkinson's disease. Brain 115: 199-210, 1992.

Barsalou LW, Huttenlocher J, Lamberts K. Basing categorization on individuals and events. Cogn Psychol 36: 203-272, 1998.

Buhusi CV, Meck WH. What makes us tick? Functional and neural mechanisms of interval timing. Nat Rev Neurosci 6: 755-765, 2005.

Buonomano DV, Karmarkar UR. How do we tell time? Neuroscientist 8: 42-51, 2002.

Cattell RB. The scree test for the number of factors. Multiv Behav Res 1: 245-276, 1966.

Drake C, Botte MC. Tempo sensitivity in auditory sequences: evidence for a multiple-look model. Percept Psychophys 54: 277-286, 1993.

Draper NR, Smith H. Applied Regression Analysis. New York: Wiley, 1981.

Fetterman JG, Killeen PR. A componential analysis of pacemaker-counter timing systems. J Exp Psychol Hum Percept Perform 16: 766-780, 1990.

Getty D. Discrimination of short temporal intervals: a comparison of two models. Percept Psychophys 18: 1-8, 1975.

Gibbon J, Malapani C, Dale CL, Gallistel C. Toward a neurobiology of temporal cognition: advances and challenges. Curr Opin Neurobiol 7: $170-184,1997$.

Goldstone S, Boardman WA, Lhamon WT. Intersensory comparison of temporal judgments. J Exp Psychol 57: 243-248, 1959.

Goldstone S, Lhamon WT. Studies of auditory-visual differences in human time judgment: 1. Sounds are judged longer than lights. Percept Mot Skills 39: 63-82, 1974.

Goodale MA, Milner AD. Separate visual pathways for perception and action. Trends Neurosci 15: 20-25, 1992.

Goodale MA, Westwood DA. An evolving view of duplex vision: separate but interacting cortical pathways for perception and action. Curr Opin Neurobiol 14: 203-211, 2004.

Grondin S. Production of time intervals from segmented and nonsegmented inputs. Percept Psychophys 52: 345-350, 1992.

Grondin S. From physical time to the first and second moments of psychological time. Psychol Bull 127: 22-44, 2001.

Grondin S, Meilleur-Wells G, Ouellette C, Macar F. Sensory effects on judgments of short-time intervals. Psychol Res 61: 261-268, 1998.

Harrington DL, Haaland KY. Neural underpinnings of temporal processing: a review of focal lesion, pharmacological, and functional imaging research. Rev Neurosci 10: 91-116, 1999.

Hazeltine E, Helmuth LL, Ivry RB. Neural mechanisms of timing. Trends Cogn Sci 1: 163-169, 1997.

Ivry RB, Hazeltine RE. Perception and production of temporal intervals across a range of durations: evidence of a common timing mechanism. $J$ Exp Psychol Hum Percept Perform 21: 3-18, 1995.

Jancke L, Loose R, Lutz K, Specht K, Shah NJ. Cortical activations during paced finger-tapping applying visual and auditory pacing stimuli. Cogn Brain Res 10: 51-66, 2000. 
Jantzen KJ, Steinberg FL, Kelso JAS. Brain networks underlying human timing behavior are influenced by prior context. Proc Natl Acad Sci USA 101: 6815-6820, 2004.

Jantzen KJ, Steinberg FL, Kelso JAS. Functional MRI reveals the existence of modality and coordination-dependent timing networks. Neuroimage 25: 1031-1042, 2005.

Kaiser HF. The application of electronic computers to factor analysis. Educ Psychol Meas 20: 141-151, 1960.

Karmarkar UR, Buonomano DV. Timing in the absence of clocks: encoding time in neural network states. Neuron 53: 427-438, 2007.

Keele S, Nicoletti R, Ivry R, Pokorny R. Do perception and motor production share common timing mechanisms? A correlational analysis. Acta Psychol 60: 173-191, 1985.

Keele S, Nicoletti R, Ivry R, Pokorny R. Mechanisms of perceptual timing: beat-based or interval-based judgments? Psychol Res 50: 251-256, 1989.

Leon MI, Shadlen MN. Representation of time by neurons in the posterior parietal cortex of the macaque. Neuron 38: 317-327, 2003.

Lewis PA, Miall RC. Distinct systems for automatic and cognitively controlled time measurement: evidence from neuroimaging. Curr Opin Neurobiol 13: 250-255, 2003.

Macar F, Coull J, Vidal F. The supplementary motor area in motor and perceptual time processing: fMRI studies. Cogn Process 7: 89-94, 2006.

Macar F, Lejeune H, Bonnet M, Ferrara A, Pouthas V, Vidal F, Maquet P. Activation of the supplementary motor area and of attentional networks during temporal processing. Exp Brain Res 142: 475-485, 2002.

Matell M, Meck WH. Neuropsychological mechanisms of interval timing behavior. Bioessays 22: 94-103, 2000.

Meck WH, Benson AM. Dissecting the brain's internal clock: how frontalstriatal circuitry keeps time and shifts attention. Brain Cogn 48: 195-211, 2002.

Meegan DV, Aslin RN, Jacobs RA. Motor timing learned without motor training. Nat Neurosci 3: 860-862, 2000.

Merchant H, Battaglia-Mayer A, Georgopoulos AP. Neural responses during interception of real and apparent circularly moving targets in motor cortex and area 7a. Cereb Cortex 14: 314-331, 2004.

Merchant H, Georgopoulos AP. Neurophysiology of perceptual and motor aspects of interception. J Neurophysiol 95: 1-13, 2006.

Merchant H, Luciana M, Hooper C, Majestic S, Tuite P. Interval timing and Parkinson's disease: heterogeneity in temporal performance. Exp Brain Res 184: 233-248, 2008.

Penney TB. Modality differences in interval timing attention, clock speed, and memory. In: Functional and Neural Mechanisms of Timing, edited by Meck WH. Boca Raton, FL: CRC Press, 2003, p. 209-234.

Pouthas V, George N, Poline JB, Pfeuty M, VandeMoorteele PF, Hugueville L, Ferrandez AM, Lehericy S, LeBihan D, Renault B. Neural network involved in time perception: an fMRI study comparing long and short interval estimation. Hum Brain Mapp 25: 433-441, 2005.

Rammsayer TH, Lima SD. Duration discrimination of filled and empty auditory intervals: cognitive and perceptual factors. Percept Psychophys 50: 565-574, 1991.

Rao SM, Harrington DL, Haaland KY, Bobholz JA, Cox RW, Binder JR. Distributed neural systems underlying the timing of movements. J Neurosci 17: 5528-5535, 1997.

Rao SM, Mayer AR, Harrington DL. The evolution of brain activation during temporal processing. Nat Neurosci 4: 317-323, 2001.

Repp BH, Penel A. Auditory dominance in temporal processing: new evidence from synchronization with simultaneous visual and auditory sequences. J Exp Psychol Hum Percept Perform 28: 1085-1099, 2002.

Robertson SD, Zelaznik HN, Lantero DA, Bojczyk KG, Spencer RM, Doffin JG, Schneidt T. Correlations for timing consistency among tapping and drawing tasks: evidence against a single timing process for motor control. J Exp Psychol Hum Percept Perform 25: 1316-1330, 1999.

Schubotz RI, Friederici AD, Yves von Cramon D. Time perception and motor timing: a common cortical and subcortical basis revealed by fMRI. Neuroimage 11: 1-12, 2000.

Schulze HH. The perception of temporal deviations in isochronic patterns. Percept Psychophys 45: 291-295, 1989.

Snedecor GW, Cochran WG. Statistical Methods. Ames, IA: Iowa State Univ. Press, 1989.

Spencer RM, Verstynen T, Brett M, Ivry R. Cerebellar activation during discrete and not continuous timed movements: an fMRI study. Neuroimage 36: 378-387, 2007

Spencer RM, Zelaznik HN. Weber (slope) analyses of timing variability in tapping and drawing tasks. J Mot Behav 35: 371-381, 2003.

Treisman M, Faulkner A, Naish PL. On the relation between time perception and the timing of motor action: evidence for a temporal oscillator controlling the timing of movement. Q J Exp Psychol A Hum Exp Psychol 45: 235-263, 1992.

Wearden JH, Edwards H, Fakhri M, Percival A. Why "sounds are judged longer than lights": application of a model of the internal clock in humans. Q J Exp Psychol B Comp Physiol Psychol 51: 97-120, 1998.

Wing AM, Kristofferson AB. Response delays and the timing of discrete motor responses. Percept Psychophys 14: 5-12, 1973.

Wright BA, Buonomano DV, Mahncke HW, Merzenich MM. Learning and generalization of auditory temporal-interval discrimination in humans. J Neurosci 17: 3956-3963, 1997

Zar JH. Biostatistical Analysis. Englewood Cliffs, NJ: Prentice Hall, 1996.

Zelaznik HN, Spencer RM, Ivry RB. Dissociation of explicit and implicit timing in repetitive tapping and drawing movements. J Exp Psychol Hum Percept Perform 28: 575-588, 2002. 